\title{
Probing the Interstellar Medium and Stellar Environments of Long-Duration GRBs
}

\author{
Miroslava Dessauges-Zavadsky ${ }^{1}$, Jason X. Prochaska ${ }^{2}$ \\ and Hsiao-Wen Chen ${ }^{3}$ \\ ${ }^{1}$ Geneva Observatory, University of Geneva, 51, Ch. des Maillettes, CH-1290 \\ Sauverny, Switzerland \\ email: miroslava.dessauges@obs .unige.ch \\ ${ }^{2}$ UCO/Lick Observatory, University of California, Santa Cruz, CA 95064, USA \\ email:xavier@ucolick.edu \\ ${ }^{3}$ Department of Astronomy, University of Chicago, 5640 S. Ellis Ave., Chicago, IL 60637, USA \\ email:hchen@oddjob.uchicago.edu
}

\begin{abstract}
We review the properties of the gas surrounding high-redshift gamma-ray bursts (GRBs) assessed through the analysis of damped Lyman-alpha systems (DLAs) identified in their afterglow spectra. These GRB-DLAs are characterized by large H I column densities with a median of $N(\mathrm{HI})=10^{21.7} \mathrm{~cm}^{-2}$, no molecular gas signatures, metallicities ranging from $1 / 100$ to nearly solar with a median exceeding $1 / 10$ solar, and no anomalous abundance patterns. The detection of the atomic $\mathrm{Mg}$ lines and the time-variability of the fine-structure lines demonstrates that the majority of the neutral gas along the GRB sightlines is located between $50 \mathrm{pc}$ and a few kpc from the GRB. This implies that this gas is presumably associated with the ambient interstellar medium of the host galaxy and that the derived properties from low-ionization lines do not directly constrain the local environment of the GRB progenitor. The highly ionized gas, traced by NV lines, which could result from a pre-existing H II region produced by the GRB progenitor and neighboring OB stars, appears on the other hand to be very local to the GRB at about $10 \mathrm{pc}$, yielding a snapshot of the medium's physical conditions at this radius.
\end{abstract}

Keywords. gamma rays: observations - galaxies: ISM - ISM: abundances

\section{Introduction}

Like quasars (QSOs), GRBs provide a bright - albeit transient - light beam. Two types of GRBs are now well established, the short and the long ones with durations $<2$ s and $>2$ s, respectively. The long-duration GRBs give rise to brighter optical afterglows and are of our interest in this work. Much of the recent advances was enabled by the launch of the Swift satellite. Thanks to its rapid and precise localization of GRBs and thanks to the 'target of opportunity' optical observations, high-quality and highresolution spectroscopy was achieved for a number of afterglows on 10-m class telescopes. Imprinted in the quasi power-law spectrum of the afterglow are the signatures of the circumstellar material from the progenitor, the H II region produced by the progenitor and neighboring OB stars, the neutral interstellar medium (ISM) of the host galaxy, any diffuse gas within the galactic halo, and finally intergalactic material located on the GRB sightline. Unfortunately, even though these phases arise at distinct distances along the sightline, the observed spectrum resolves only the relative velocities of the gas. Therefore, for the analysis, one has to keep in mind that all these phases are potentially mixed in the afterglow spectrum.

It is expected that GRBs originate in star-forming regions and have massive stars as progenitors. Several observational evidences point toward this hypothesis. At low redshift, 


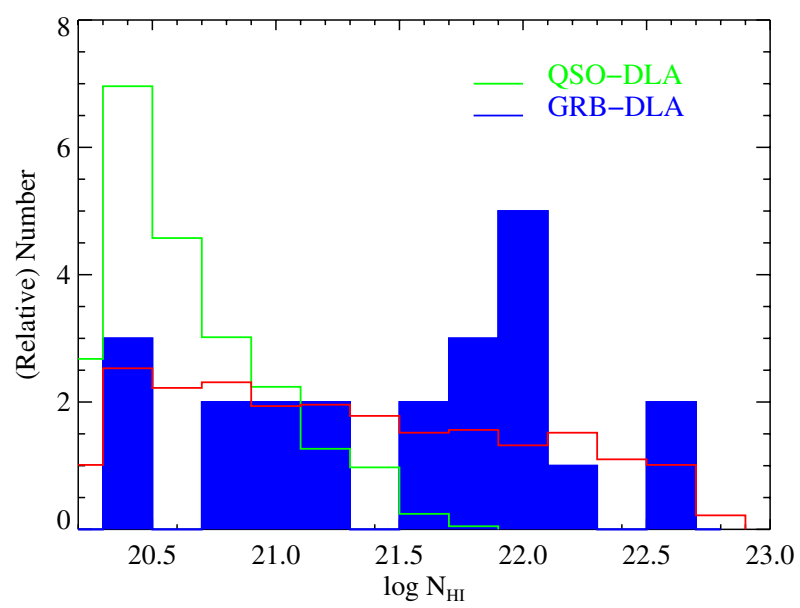

Figure 1. Distributions of H I column densities for both GRB-DLA (filled, solid histogram) and QSO-DLA (solid histogram). The GRB-DLA have a median value $\log N(\mathrm{HI})=21.7$, which exceeds all but a few QSO-DLA observed to date.

the association between the GRBs and massive star progenitors is well established by (i) Type Ic supernovae (SNe) identified at the positions of GRB events (Hjorth et al. 2003; Mirabal et al. 2006) and (ii) the location of GRBs in Wolf-Rayet galaxies (Hammer et al. 2006). At high redshift, the connection between GRBs and star-forming regions is inferred by (i) GRBs found in blue galaxies with nebular lines indicative of on-going star formation (Le Floc'h et al. 2003; Prochaska et al. 2004) and (ii) GRBs observed within a few kpc from the weighted flux centroid of their host (Fruchter et al. 2006).

What are the properties of the ISM within and near star-forming regions in the young Universe? What physical constraints does one place on the GRB surrounding gas (metallicity, density, temperature, dust and molecular content)? Where is the gas that we observe? Can we probe gas very local to the progenitor? These are the questions that motivate our work. Our GRB sample is composed of only afterglow spectra with (i) high signal-to-noise ratios and sufficient spectral resolution $(R>2000)$ to allow accurate column density measurements, and (ii) the presence of damped Ly $\alpha$ lines $\left(N(\mathrm{HI}) \geqslant 2 \times 10^{20}\right.$ $\mathrm{cm}^{-2}$ ) or of strong low-ionization lines. In total, our sample contains 16 afterglow spectra of GRB-DLA at redshifts between 1 and 6.5.

\section{Neutral gas}

\subsection{HI content: Fuel for star formation}

Figure 1 shows the distributions of H I column densities for both GRB-DLA and QSODLA. The GRB-DLA have large H I column densities, with a median value of $\log N(\mathrm{HI})=$ 21.7 (Prochaska et al. 2007). Out of about 1000 such measurements for QSO-DLA, not a single has $N(\mathrm{HI}) \geqslant 10^{22} \mathrm{~cm}^{-2}$. This difference in $N(\mathrm{HI})$ between GRB-DLA and QSODLA can be naturally explained by sightline geometries: the GRB sightlines originate within the host galaxy and very certainly within a young star-forming region, while the QSO sightlines pass randomly through the galaxy and statistically more often through the outer regions, because of the higher cross-sections, and have lower $N(\mathrm{HI})$.

Massive stars are observed to form in molecular clouds, so we may wonder whether the high GRB-DLA H I column densities are consistent with that predicted for GRBs embedded within molecular clouds. Three observational results do not support the 


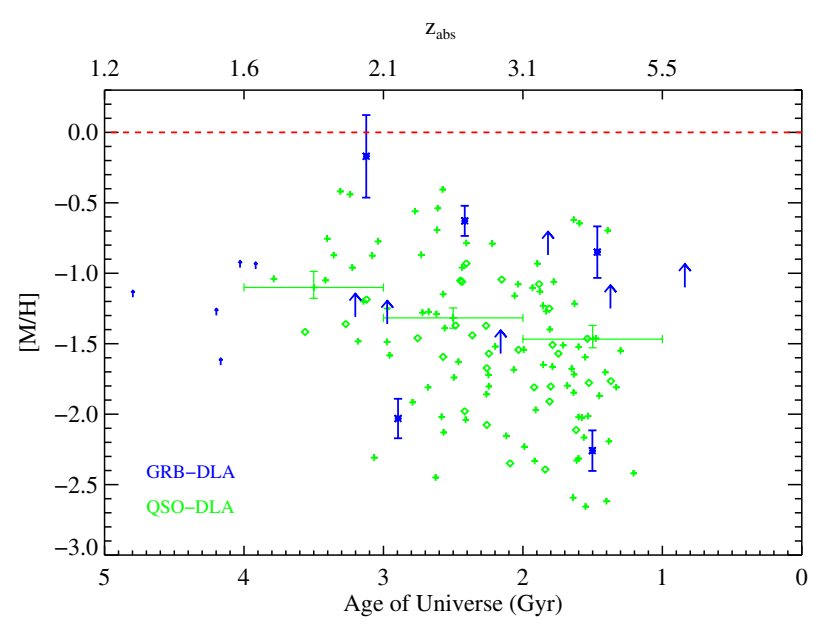

Figure 2. Metallicity $[\mathrm{M} / \mathrm{H}]$ measurements (we considered the metal-lines which are unsaturated, so for most measurements $[\mathrm{M} / \mathrm{H}]=[\mathrm{S} / \mathrm{H}]$ ) for GRB-DLA (large symbols and arrows) and for QSO-DLA (small symbols) as a function of the age of the Universe. The GRB-DLA distribution spans two orders of magnitude and has a median value that exceeds $1 / 10$ solar and is higher than the cosmic mean metallicity of H I in the ISM of QSO-DLA (large crosses).

presence of molecular gas: (i) there are too many GRB-DLA with H I column densities lower than $10^{21.5} \mathrm{~cm}^{-2}$ compared to the Galactic molecular clouds (Jakobsson et al. 2006); (ii) among the 5 sightlines for which $\mathrm{H}_{2}$ lines are covered, none shows molecular line detection and sets a very low molecular fraction $f\left(\mathrm{H}_{2}\right)<10^{-6}$ (Tumlinson et al. 2007); and (iii) low $\mathrm{C}^{0} / \mathrm{C}^{+}$ratios $\left(<10^{-4}\right)$ are observed favoring gas dominated by a warm, less dense phase (Prochaska et al. 2007).

As a first result, we can conclude that the observed H I gas traces more likely the ambient ISM gas, instead of gas associated with the GRB dense molecular cloud.

\subsection{Metallicity}

In the collapsar model, a large angular momentum is required to power the GRB. Since high-metallicity stars are expected to have significant mass-loss by winds, leading to the loss of their angular momentum, this suggests that GRB progenitors should have metallicities less than 1/10 solar (Hirschi et al. 2005; Langer \& Norman 2006; Woosley \& Heger 2006). The observations of GRB hosts at $z<1$ do seem to show a bias toward lowmetallicities. Indeed, the few metallicities derived from the nebular line analysis point to sub-solar metallicities (Sollerman et al. 2005). The GRB hosts also generally have sub- $\mathrm{L}_{*}$ luminosities (Le Floc'h et al. 2003; Fruchter et al. 2006), consistent with low metallicities. Modjaz et al. (2008) has, in addition, shown that GRBs occur in lower metallicity galaxies than the ones hosting a random sample of Type Ic SN. If this is confirmed, it would offer compelling evidence that metallicity plays a role in the GRB progenitors.

What about the metallicities in GRB hosts at high redshift $(z>2)$ ? Figure 2 shows the measured GRB-DLA metallicities as a function of the age of the Universe and compared with the QSO-DLA metallicities. GRB-DLA exhibit a large spread of metallicities ranging from 1/100 solar to nearby solar, with a median value of $[\mathrm{M} / \mathrm{H}]$ exceeding $1 / 10$ solar (Prochaska et al. 2007). Almost all the GRB-DLA metallicities are higher than the cosmic mean metallicity of H I in the ISM of QSO-DLA. This has two main implications. First, the gas near star-forming regions seems to have an enhanced metallicity. This can be a 
result of metallicity gradients within the galaxies, since as we have previously pointed out GRB-DLA more likely probe the inner regions of galaxies and QSO-DLA the outer ones. Second, there is little evidence that GRBs at high-redshift prefer low metallicities, i.e. lower than $1 / 10$ solar.

\subsection{Chemical properties}

We have used the $\mathrm{Zn} / \mathrm{Fe}$ ratio to examine the dust content in the GRB-DLA. They do show uniformly large $[\mathrm{Zn} / \mathrm{Fe}]$ ratios $(>0.5 \mathrm{dex})$, suggesting a highly depleted gas with more than $50 \%$ of Fe locked into dust grains. This is further supported by the sub-solar [Ti/Fe] ratios (Dessauges-Zavadsky et al. 2002). However, as pointed out by Savaglio (2006), the depletion pattern resembles more likely the warm Galactic ISM rather than the cold, dense clouds one associated with proto-molecular clouds.

The GRB-DLA show large $[\alpha / \mathrm{Fe}]$ ratios, exceeding 0.5 dex and being systematically larger than in QSO-DLA. Is this $\alpha$-enhancement a result of nucleosynthesis or dust depletion effects? On the nucleosynthetic point of view, an $\alpha$-enhancement is expected, first because the GRB progenitors certainly are massive stars and second because high specific star formation rates are observed in the host galaxies implying young ages compared to the timescale for Type Ia SN enrichment. The contribution from differential dust depletion effects can be estimated to $\sim 0.3$ dex. This leaves place for a remaining $\alpha$-enhancement larger than 0.3 dex, reflecting enrichment by massive stars and being consistent with GRBs tracing star formation.

In summary, the GRB-DLA have a median metallicity larger than 1/10 solar, they show important dust depletion levels but still lower than those of cold/dense clouds, and they do not show any anomalous abundance patters which could occur if the gas represented partially mixed SN ejecta or circumstellar material. As a second result, we conclude that the derived metal abundances trace more likely the ambient ISM near the GRBs rather than the GRB circumstellar material.

\section{Distance diagnostics of the neutral gas}

Two distance diagnostics allow us to estimate the distance of the observed neutral gas. First, the detection of the atomic Mg lines. Indeed, we do observe large column densities of $\mathrm{Mg}^{0}\left(\approx 10^{14.7} \mathrm{~cm}^{-2}\right)$ with $\mathrm{Mg}$ I lines coincident in velocity with other low-ionization lines. With a very low ionization potential of $7.7 \mathrm{eV}$, this neutral gas is not shielded by the large $\mathrm{HI}$ columns, and therefore $\mathrm{Mg}^{0}$ is fully ionized in less than $1000 \mathrm{~s}$ if the gas is located within $50 \mathrm{pc}$ of the GRB. As a consequence, the detection of Mg I places the gas at more than $50 \mathrm{pc}$ from the GRB.

Second, the transitions from the excited states of $\mathrm{C}^{+}, \mathrm{Si}^{+}, \mathrm{O}^{0}, \mathrm{Fe}^{+}$, and $\mathrm{Ni}^{+}$. They appear to be generic features in the GRB afterglow spectra, with their absorption lines being coincident in velocity with the low-ionization lines. These transitions have not been detected in QSO-DLA so far. They have been observed in environments characterized by extreme densities and temperatures, such as the Broad Absorption Line quasars, $\eta$ Carina and $\beta$ Pictoris. Three excitation mechanisms allow to produce these fine-structure lines: indirect UV pumping, direct IR pumping, and collisional excitation. Prochaska et al. (2006) demonstrated that the UV pumping mechanism dominates out to $1 \mathrm{kpc}$. This has two main implications. First, the gas traced by the fine-structure lines is not a highdensity circumstellar gas, and second the UV-pumping predicts line variability due to the decline of the afterglow ionizing flux as a function of time. The fine-structure line variability has been reported in two GRBs so far, GRB 020813 (Dessauges-Zavadsky et al. 


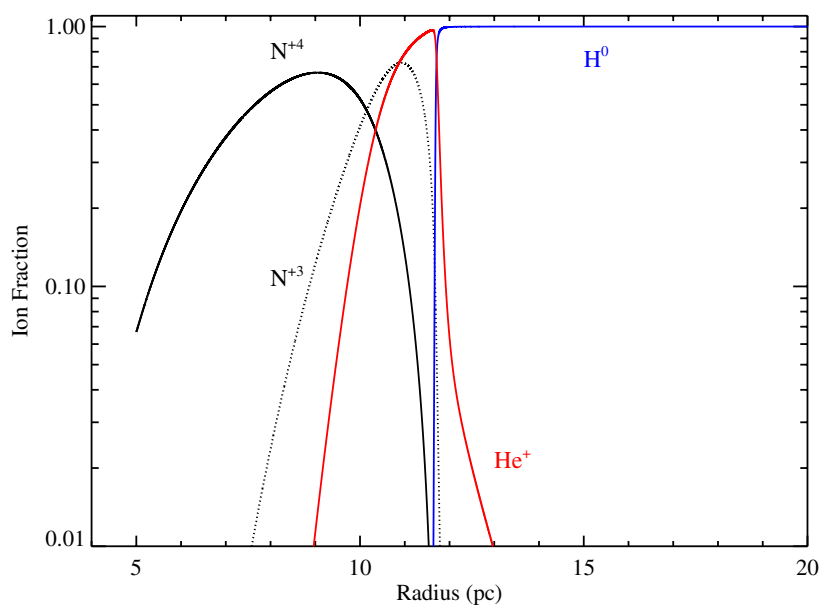

Figure 3. Ion fractions as a function of distance from the GRB afterglow. The calculation assumes a constant density medium $\left(n_{\mathrm{H}}=10 \mathrm{~cm}^{-3}\right)$ and the total photon flux from an afterglow integrated from $t=+10 \mathrm{~s}$ to $+1000 \mathrm{~s}$ in the observer frame. The distance to the $\mathrm{N}^{4+}$ maximum ionization fraction is of about $10 \mathrm{pc}$.

2006) and GRB 060418 (Vreeswijk et al. 2007). These detections allow to constrain the distance of the neutral gas between $100 \mathrm{pc}$ and $1.5 \mathrm{kpc}$.

In summary and as a third result, the $\mathrm{Mg}$ I and fine-structure transitions demonstrate that the majority of neutral gas along the GRB sightline is located between $50 \mathrm{pc}$ and a few $\mathrm{kpc}$ from the GRB. This is presumably beyond the immediate star-forming region of the GRB progenitor and therefore one associates this gas with the ambient ISM of the host galaxy. This implies that analysis related to the low-ionization transitions, e.g. metallicity measurements, chemical studies, does not directly constrain the local environment of the GRB progenitor.

\section{Ionized gas}

What have we learnt so far? The detected neutral gas in afterglow spectra traces the ambient ISM with most of this gas lying between $100 \mathrm{pc}$ and $1.5 \mathrm{kpc}$ from the GRB. There is no evidence for molecular gas, despite the observed large H I surface densities and the presence of dust. What about the ionized gas which may be present in pre-existing H II regions produced by the GRB progenitor and neighboring OB stars?

A number of GRB sightlines exhibit X-ray absorption with implied metal column densities that significantly exceed the neutral ISM column densities measured from optical spectra (Watson et al. 2007, Galama \& Wijers, 2001). These observations provide hints for a large reservoir of highly ionized gas near the GRB. Therefore, we have initiated a survey for N V lines. Why NV? It is because $\mathrm{N}^{3+}$ has a high ionization potential $(\mathrm{IP}=77 \mathrm{eV})$ that makes the production of $\mathrm{N}^{4+}$ difficult, especially using stellar radiation fields. $\mathrm{N} \mathrm{V}$ is believed to trace collisionally ionized gas either in equilibrium at a high temperature $\left(T>10^{5} \mathrm{~K}\right)$ or out of equilibrium due to a post-shocked gas cooling from $T>10^{6} \mathrm{~K}$.

We identify a high incidence (6/7) of spectra exhibiting N V gas (Prochaska et al. 2008). The N V lines show the following characteristics: (i) high detection rates; (ii) large optical depths with large integrated column densities $N\left(\mathrm{~N}^{4+}\right) \gtrsim 10^{14} \mathrm{~cm}^{-2}$; (iii) narrow profiles $\left(\lesssim 50 \mathrm{~km} \mathrm{~s}^{-1}\right)$; and (iv) small velocity offset from low-ionization and fine-structure lines 
$\left(\delta v \lesssim 20 \mathrm{~km} \mathrm{~s}^{-1}\right)$. These characteristics are unlike the $\mathrm{NV}$ gas observed in the halo and disk of the Milky Way and high-redshift QSO-DLA, but best resemble the narrow absorption lines associated with QSOs and $z \sim 3$ starburst galaxies.

Scenarios related to material shock-heated by the progenitor's stellar wind are not the dominant mechanisms for producing the observed $\mathrm{N}^{4+}$ absorption, since then one would expect velocity offsets of the ionized material relatively to the neutral gas by several thousands of $\mathrm{km} \mathrm{s}^{-1}$ (Starling et al. 2005; van Marle et al. 2005). The swept up ISM material by the stellar wind should on the other hand almost coincide in velocity with the neutral gas, but there the gas rapidly cools to $\approx 10^{4} \mathrm{~K}$ which is not sufficient to produce $\mathrm{N}^{4+}$ (Lamers \& Cassinelli 1999). As a consequence, the GRB afterglow ionizing flux appears as the natural mechanism to produce kinematically cold N V lines, i.e. with no offset from the neutral gas and with narrow profiles. Figure 3 shows that the afterglow will photoionize nitrogen to $\mathrm{N}^{4+}$ at $r \approx 10 \mathrm{pc}$. Within the photoionization scenario, the observations imply the progenitor's stellar wind is confined to $r<10 \mathrm{pc}$. This places strong constraints on the GRB progenitor and/or the ISM material close to the GRB: low mass-loss rates, short stellar lifetimes and/or high ISM densities of $n \geqslant 10^{3} \mathrm{~cm}^{-3}$ are required.

As a fourth result, we conclude that the highly ionized $\mathrm{N} V$ gas detected in the afterglow spectra traces the very local gas to the GRB at about $10 \mathrm{pc}$, yielding a snapshot of the medium's physical conditions at this radius.

\section{References}

Dessauges-Zavadsky, M., Prochaska, J. X., \& D'Odorico, S. 2002, A\&A, 391, 801

Dessauges-Zavadsky, M., Chen, H.-W., Prochaska, J. X., et al. 2006, ApJ, 648, L89

Fruchter, A. S., Levan, A. J., Strolger, L., et al. 2006, Nature, 441, 463

Galama, T. J. \& Wijers, R. A. M. J. 2001, ApJ, 459, L209

Hammer, F., Flores, H., Schaerer, D., et al. 2006, A\&SA, 454, 103

Hirschi, R., Meynet, G., \& Maeder, A. 2005, A\&A, 443, 581

Hjorth, J., Sollerman, J., Moller, P., et al. 2003, Nature, 423, 847

Jakobsson, P., Fynbo, J. P.U., Ledoux, C., et al. 2006, A\&SA, 460, L13

Lamers, H. J. G. L. M. \& Cassinelli, J. P. 1999, Introduction to Stellar Winds (Cambridge: CUP)

Langer, N. \& Norman, C. A. 2006, ApJ, 638, L63

Le Floc'h, E., Duc, P.-A., Mirabel, I. F., et al. 2003, A\&A, 400, 499

Modjaz, M., Kewley, L., Kirshner, R. P., et al. 2008, AJ, 135, 1136

Mirabal, N., Halpern, J. P., An, D., et al. 2006, ApJ, 643, L99

Prochaska, J. X., Bloom, J. S., \& Chen, H.-W. et al. 2004, ApJ, 611, 200

Prochaska, J. X., Chen, H.-W., \& Bloom, J. S. 2006, ApJ, 648, 95

Prochaska, J. X., Chen, H.-W., Dessauges-Zavadsky, M., \& Bloom, J. S. 2007, ApJ, 666, 267

Prochaska, J. X., Dessauges-Zavadsky, M., Raminez-Ruiz, E. \& Chen, H. W. 2008, ApJ, in press

Savaglio, S. 2006, New Journal of Phys., 8, 195

Sollerman, J., Östlin, G., Fynbo, J. P. U., et al. 2005, NewA, 11, 103

Starling, R. L. C., Wijers, R. A. M. J., Hughes, M. A., et al. 2003, MNRAS, 360, 305

Tumlinson, J., Prochaska, J. X., Chen, H.-W., et al. 2007, ApJ, 668, 667

van Marle, A. J., Langer, N., \& Gracía-Segura, G. 2005, A\& $A$, 444, 837

Vreeswijk, P. M., Ledoux, C., Smette, A., et al. 2007, A\&GA, 468, 83

Watson, D., Hjorth, J., Fynbo, J. P. U., et al. 2007, ApJ, 660, L101

Woosley, S. E. \& Heger, A. 2006, ApJ, 637, 914 